\title{
Winning combinations of history-dependent games
}

\author{
Roland J. Kay 円 and Neil F. Johnson \\ Physics Department, Oxford University, Parks Road, Oxford, OX1 3PU, U.K.
}

(Dated: October 28, 2018)

\begin{abstract}
The Parrondo effect describes the seemingly paradoxical situation in which two losing games can, when combined, become winning [Phys. Rev. Lett. 85, 24 (2000)]. Here we generalize this analysis to the case where both games are history-dependent, i.e. there is an intrinsic memory in the dynamics of each game. New results are presented for the cases of both random and periodic switching between the two games.
\end{abstract}

\section{INTRODUCTION}

The Parrondo effect [1] is the counter-intuitive situation whereby individually losing games somehow 'cooperate' to produce a winning game. In particular, these losing games can be combined randomly and yet the effect still emerges. The intriguing aspect is that randomness in this system is acting in a constructive way. Possible applications of this effect have been suggested in several fields including biogenesis [2], molecular transport [3] [4], random walks [5] and biological systems [6]. Even in the social sciences, 'winning' models for investment have been reported $[7$.

Consider a gambling game in which the player has a time-dependent capital $X(t)$ where $t=0,1,2, \ldots$, and whose evolution is determined by tossing biased coins. The rules as to which coins to toss, and hence the probability of winning, are determined by the history, i.e. the game is history-dependent. The game can be divided into three regimes: winning, losing and fair (for which $\langle X(t)\rangle$ is respectively an increasing, decreasing or constant function of $t$ ). Parrondo et al [1] considered combinations of such a history-dependent game $\mathrm{B}$, as described above, and a simple biased coin toss (i.e. game A which is history-independent and hence has no memory). In Parrondo et al's study, game A is defined by the probability $p$ of $X(t)$ increasing, where $p=\frac{1}{2}-\epsilon$. Hence game $\mathrm{A}$ is a losing game for $\epsilon>0$. Game $\mathrm{B}$ is defined by the probabilities of four biased coins: $\left\{p_{1}, p_{2}, p_{3}, p_{4}\right\}$. The particular coin played at a given time step depends upon the history of the game as shown in Table [1. Parrondo et al showed that two losing games $\mathrm{A}$ and $\mathrm{B}$ can be combined to yield a winning game, if the games are alternated either periodically or at random.

The reason that Parrondo's paradox arises for combined A-B games is that losing cycles in game B are effectively broken up by the memoryless behavior, or 'noise', of game A. The question therefore arises: what happens if both games are of type B, and hence have losing cycles? Can the losing cycle in one game break up the losing cycle in the other in order to produce 'winning dynamics'? Since the answer is not obvious, and since

*Electronic address: roland.kay@physics.ox.ac.uk

\begin{tabular}{ccccc}
\hline \hline Time step & Time step & Coin & Prob. of win & Prob. of loss \\
$t-2$ & $t-1$ & at $t$ & at $t$ & at $t$ \\
\hline Loss & Loss & $B_{1}$ & $p_{1}$ & $1-p_{1}$ \\
Loss & Win & $B_{2}$ & $p_{2}$ & $1-p_{2}$ \\
Win & Loss & $B_{3}$ & $p_{3}$ & $1-p_{3}$ \\
Win & Win & $B_{4}$ & $p_{4}$ & $1-p_{4}$ \\
\hline \hline
\end{tabular}

TABLE I: Type B games consist of four coins. The coin to be played at time step $t$ is determined by the results of the previous two time steps, as shown.

the Parrondo effect promises to have a variety of applications, it is important to establish whether two historydependent games will indeed produce a Parrondo effect. This provides the motivation for the present study.

In this paper, we generalize the analysis of Ref. [1] to the case where both games are history-dependent, i.e. there is an intrinsic memory in the dynamics of each game. We find specific regimes which do indeed exhibit a Parrondo effect. New results are presented for the cases of both random and periodic switching between the two games. The paper is organized as follows. In Sec. II we investigate random combinations of two games of type B. In Sec. III we consider periodic combinations of such games. In Sec. IV we investigate the effect of varying the switching probability. Section V provides a summary.

\section{RANDOM COMBINATIONS OF HISTORY-DEPENDENT GAMES}

We now extend the analysis of Parrondo et al to the case of two history-dependent games of type B. We define $\left\{p_{i}-\epsilon\right\}$ and $\left\{q_{i}-\epsilon\right\}$ as the probability sets defining the $\mathrm{B}$ games and $\left\{r_{i}-\epsilon\right\}$ as the probability set defining the combined game. We follow Parrondo et al in only considering losing games which result by subtracting a small quantity $\epsilon$ from each of the probabilities that define a fair game. As in Ref. [1], we can define a vector Markovian process $Y(t)$ based on the capital $X(t)$ as follows:

$$
Y(t)=\left(\begin{array}{c}
X(t)-X(t-1) \\
X(t-1)-X(t-2)
\end{array}\right)
$$


$Y(t)$ can take four values $( \pm 1, \pm 1)$. We label the four states of $Y(t)$ as shown in Table III.

\begin{tabular}{cc}
\hline \hline$Y(t)$ & State \\
\hline$(-1,-1)$ & 1 \\
$(+1,-1)$ & 2 \\
$(-1,+1)$ & 3 \\
$(+1,+1)$ & 4 \\
\hline \hline
\end{tabular}

TABLE II: Labels for the four possible states of the Markovian process $Y(t)$, where $Y(t)$ is defined in terms of the capital $X(t)$ as prescribed by Eq. (11).

For $\epsilon=0$ both $\mathrm{B}$ games must be fair. This is achieved by the condition $\left(1-p_{4}\right)\left(1-p_{3}\right)-p_{1} p_{2}=0$ [1]. This yields the first two conditions in Eq. (2). For the combined game to be winning, we obtain the final condition listed in Eq. (2):

$$
\begin{aligned}
\left(1-p_{4}\right)\left(1-p_{3}\right) & =p_{1} p_{2} \\
\left(1-q_{4}\right)\left(1-q_{3}\right) & =q_{1} q_{2} \\
\left(1-r_{4}\right)\left(1-r_{3}\right) & <r_{1} r_{2} .
\end{aligned}
$$

If the two B games are combined randomly, the probability set for the combined game is given by:

$$
r_{i}=\alpha p_{i}+(1-\alpha) q_{i}
$$

where $\alpha$ is the probability that the game characterized by $\left\{p_{i}\right\}$ will be chosen. We will typically take $\alpha=\frac{1}{2}$. The third condition in Eq. (2) now becomes:

$$
\left(2-p_{4}-q_{4}\right)\left(2-p_{3}-q_{3}\right)<\left(p_{1}+q_{1}\right)\left(p_{2}+q_{2}\right) .
$$

Given that we require the initial games to be fair for $\epsilon=0$, we can use the first two conditions in Eq. (2) to substitute for $p_{1}$ and $q_{1}$. Hence:

$$
\begin{aligned}
& \left(2-p_{4}-q_{4}\right)\left(2-p_{3}-q_{3}\right)< \\
& \left(\frac{\left(1-p_{4}\right)\left(1-p_{3}\right)}{p_{2}}+\frac{\left(1-q_{4}\right)\left(1-q_{3}\right)}{q_{2}}\right)\left(p_{2}+q_{2}\right) .
\end{aligned}
$$

\section{A. Special case $p_{2}=p_{3}, q_{2}=q_{3}$}

In order to reduce the number of free variables so that the different regions of the parameter space can be displayed in a three dimensional figure, Parrondo et al [1] made the restriction $p_{2}=p_{3}$. Here we are going to reduce the number of free variables by appealing to the first two conditions in Eq. (2). These conditions give $p_{1}$ and $q_{1}$ in terms of $\left\{p_{j}\right\},\left\{q_{j}\right\} \quad(j=2,3,4)$ such that both games are fair when $\epsilon=0$.

We choose a particular game $\left\{p_{i}\right\}$ and then plot the regions in the parameter space $\left(q_{2}, q_{3}, q_{4}\right)$ which enclose all games $\left\{q_{i}\right\}$ for which the Parrondo effect is observed. Initially we treat the special case introduced by Parrondo et al using the parameter space $\left(q_{2}=q_{3}, q_{4}\right)$, taking for the first $\mathrm{B}$ game:

$$
\left\{p_{i}\right\}=\left\{\frac{9}{10}, \frac{1}{4}, \frac{1}{4}, \frac{7}{10}\right\}
$$

Rearranging Eq. (2) gives:

$$
q_{1}=\frac{\left(1-q_{4}\right)\left(1-q_{3}\right)}{q_{2}} .
$$

$q_{1}$ is a probability and is thus subject to the restriction $0<q_{1}<1$. Therefore in order to be physically realized, the game $\left\{q_{i}\right\}$ must be restricted as follows:

$$
q_{4}>1+\frac{q_{2}}{q_{3}-1}
$$

and hence, in the special case where $q_{3}=q_{2}$ :

$$
q_{4}>1+\frac{q_{2}}{q_{2}-1}
$$

From Eq. (5) the condition that defines the regions of the parameter space in which two fair games combine to yield a winning game is given by:

$$
q_{4} \begin{cases}>1+\frac{\left(p_{4}-1\right)}{p_{2}} q_{2} & \text { if } q_{2}>p_{2} \\ <1+\frac{\left(p_{4}-1\right)}{p_{2}} q_{2} & \text { if } q_{2}<p_{2}\end{cases}
$$

Figure1 depicts the regions of parameter space defined by Eqs. (10) and (9) for $\left\{p_{i}\right\}$ given by Eq. (6). In particular, Fig. 1 shows the region in which two fair games combine to yield a winning game. This is equivalent to the region in which two losing games combine to yield a winning game for some value of $\epsilon>0$. In the appendix we derive an expression for the maximum value of $\epsilon$ for which this remains true, $\epsilon_{\max }$.

Figure 2 shows $\epsilon_{\max }$, given by Eq. (A6) using the same game set $\left\{p_{i}\right\}$ as in Fig. 11. The value of $\epsilon_{\max }$ is shown for all possible games $\left\{q_{i}\right\}$ given $\left\{p_{i}\right\}$ defined by Eq. (6). This plot demonstrates the robustness of the Parrondo effect in the present case of two history-dependent games.

\section{B. General case $p_{2} \neq p_{3}, q_{2} \neq q_{3}$}

Now we drop Parrondo et al's restriction to $p_{2}=p_{3}$ and treat the general case. From Eq. (5):

$$
q_{4} \begin{cases}<1+\frac{\left(p_{4}-1\right)}{p_{2}} q_{2} & \text { if } q_{3}<1+\frac{p_{3}-1}{p_{2}} q_{2} \\ >1+\frac{\left(p_{4}-1\right)}{p_{2}} q_{2} & \text { if } q_{3}>1+\frac{p_{3}-1}{p_{2}} q_{2} .\end{cases}
$$

Figure 3 depicts the regions of parameter space defined by Eq. (11) and Eq. (8) for $\left\{p_{i}\right\}$ given by Eq. (6). Equation (11) defines two regions (labelled "I" and "II" in Fig. 3). Equation (8) excludes almost all of region I 


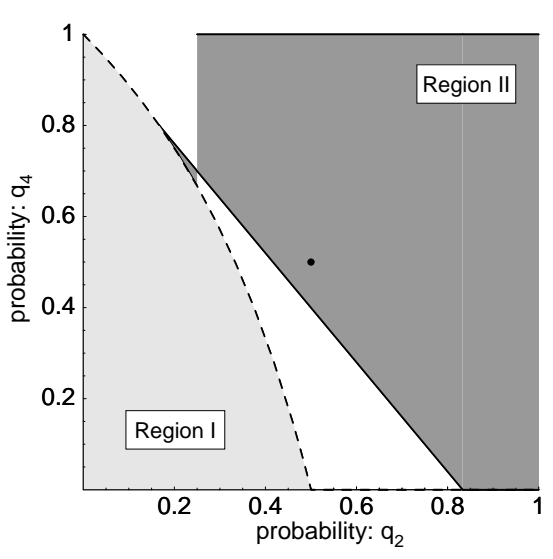

FIG. 1: Parameter space for combination of two games in the special case $p_{2}=p_{3}, q_{2}=q_{3}$ with $\left\{p_{i}\right\}$ given by Eq. (6). Region I depicts the area forbidden by Eq. (9). Region II depicts the area where the two games combine to yield a winning game. The white region in between represents the area excluded by Eq. 10, in which two fair games combine to yield a losing game. The black dot represents the parameters for Parrondo et al 's original game as described in Ref. [1] for which $\left\{q_{1}=\frac{1}{2}, q_{2}=\frac{1}{2}, q_{3}=\frac{1}{2}, q_{4}=\frac{1}{2}\right\}$.

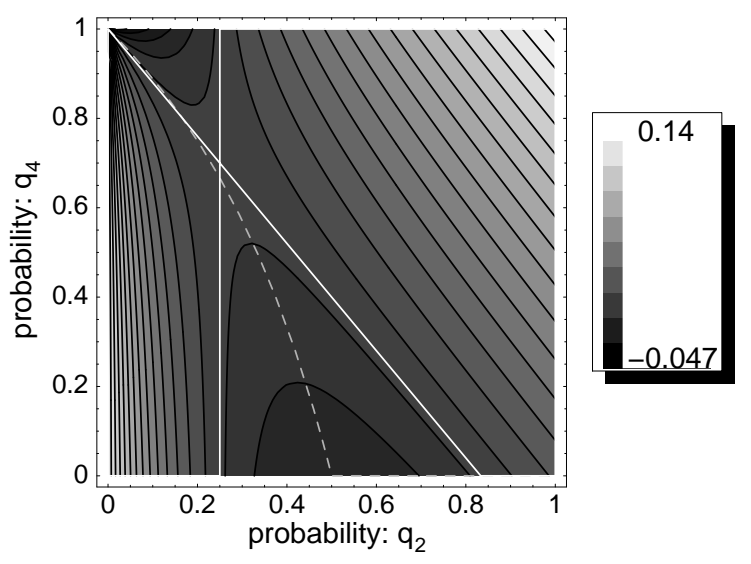

FIG. 2: Plot of the maximum value of $\epsilon$ for which the two losing games $\left\{p_{i}\right\}$ defined by Eq. (6) and $\left\{q_{1}, q_{2}, q_{2}, q_{4}\right\}$, combine to yield a winning game. The white lines indicate the locus of points where $\epsilon_{\max }=0$. The regions in which $\epsilon_{\max }<0$ correspond to two winning games combining to yield a losing game. The region to the left of the dashed line is that excluded by Eq. (9), as in Fig. 1.

in this case. An expression for $\epsilon_{\max }$ in the general case is derived in the appendix, Eq. (A7), given that $\left\{q_{i}\right\}$ is a fair game for $\epsilon=0$. In principle we could plot this over the $3 \mathrm{D}$ axes of Fig. 3. This would be the generalization of Fig. 2.

The original combination of game $\mathrm{A}$ and game $\mathrm{B}$ due to Parrondo et al (see Section I) represents a special case of our more general treatment. The game considered in Ref. [1] corresponds to combining $\left\{p_{i}\right\}$ as defined by Eq.

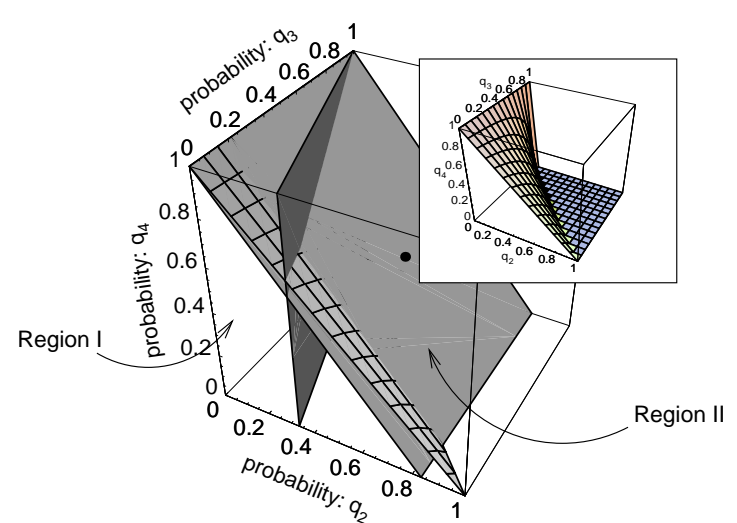

FIG. 3: Regions of parameter space in which two fair games combine to yield a winning game for $\left\{p_{i}\right\}$ given by Eq. (6). The planes indicate the boundaries of these regions (themselves marked "I" and "II"). The unmarked regions are those in which the opposite effect occurs. The surface (and the inset) show the boundary of the region forbidden by Eq. (8). The black dot represents Parrondo et al 's original game as described in Ref. [i] for which $\left\{q_{1}=\frac{1}{2}, q_{2}=\frac{1}{2}, q_{3}=\frac{1}{2}, q_{4}=\frac{1}{2}\right\}$.

(6) with $\left\{q_{1}=\frac{1}{2}, q_{2}=\frac{1}{2}, q_{3}=\frac{1}{2}, q_{4}=\frac{1}{2}\right\}$. In Figures 1 and 3 the black dot represents Parrondo et al 's original game. In both cases it can be seen to lie in the region where two losing games combine to yield a winning game.

Thus, we have derived expressions, Eqs. (10) and (11), for the region of the parameter space in which the Parrondo effect is observed to occur in the case of historydependent games being combined with equal probability $\left(\alpha=\frac{1}{2}\right)$. We have also derived expressions for the robustness of the effect, Eqs. (A6) and A7).

\section{PERIODIC COMBINATIONS OF HISTORY-DEPENDENT GAMES}

Next we investigate periodic combinations of games. Rather than randomly selecting the game to be played at each time step, game $\left\{p_{i}\right\}$ is played $a$ times and then game $\left\{q_{i}\right\}$ is played $b$ times. This cycle is repeated periodically. Figure 4 shows the capital after 500 times steps, resulting from a combination of two games for a range of values of $a$ and $b$. The capital is greater if the games are switched more frequently, as found by Parrondo et al for the combination of a simple game A and a historydependent game B. The analysis for the periodic case is more complex than for the random case because we can no longer appeal to a single game formed from a weighted average of two games.

Let the elements of the vector $\mathbf{u}_{i}$, labelled $u_{i ; j}$, be the probability of the game being in state $j$ at time $t=i$. The evolution of the game from $\mathbf{u}_{i}$ to $\mathbf{u}_{i+a+b}$ can be described 


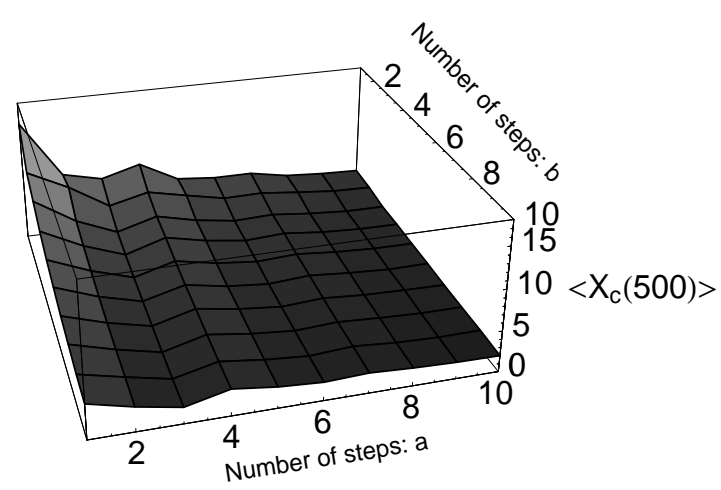

FIG. 4: Value of capital after 500 games averaged over an ensemble of $5 \times 10^{5}$ runs. Games $\left\{p_{i}\right\}$ (defined by Eq. (6)) and $\left\{q_{i}\right\}=\left\{\frac{2}{5}, \frac{3}{5}, \frac{3}{5}, \frac{2}{5}\right\}$ are combined periodically for $\epsilon=0$. Game $\left\{p_{i}\right\}$ is played $a$ times, then game $\left\{q_{i}\right\}$ is played $b$ times and so on.

as follows:

$$
\begin{gathered}
\mathbf{u}_{i+a+b}=\mathbf{B}^{b} \mathbf{A}^{a} \mathbf{u}_{i} \\
\mathbf{A}=\left(\begin{array}{cccc}
1-p_{1} & 0 & 1-p_{3} & 0 \\
p_{1} & 0 & p_{3} & 0 \\
0 & 1-p_{2} & 0 & 1-p_{4} \\
0 & p_{2} & 0 & p_{4}
\end{array}\right) \\
\mathbf{B}=\left(\begin{array}{cccc}
1-q_{1} & 0 & 1-q_{3} & 0 \\
q_{1} & 0 & q_{3} & 0 \\
0 & 1-q_{2} & 0 & 1-q_{4} \\
0 & q_{2} & 0 & q_{4}
\end{array}\right)
\end{gathered}
$$

Clearly this is not a homogeneous Markovian process because the transition matrix is not time-independent.

In order to proceed we define a homogeneous Markovian process described by the transition matrix $\mathbf{T}_{0}=$ $\mathbf{B}^{b} \mathbf{A}^{a}$ with time steps $t=(a+b) i$, where $i=0,1,2,3, \ldots$. Consider a large ensemble of games described by Eq. (12) in the long time limit. Select one of these games at random. The stationary state $\mathbf{S}_{0}$ of the homogeneous game defined by $\mathbf{T}_{0}$ gives the probability that the selected game will be in each of the four possible states (see Table III) at times $t=(a+b) i$, where $i=0,1,2,3 \ldots$. This stationary state is given by the solution to the equation $\mathbf{S}_{0}=\mathbf{T}_{0} \mathbf{S}_{0}$. Now we define a new homogeneous process, $\mathbf{T}_{1}$, by cyclically permuting the matrices in $\mathbf{T}_{0}$ once to the right (e.g. if $\mathbf{T}_{0}=\mathbf{B A A}$, then $\mathbf{T}_{1}=\mathbf{A B A}$ ). The stationary state of this process gives the probabilities of finding the game, selected from the ensemble, in each of the four possible states at times $t=(a+b) i+1$, where $i=0,1,2,3 \ldots$

In the general case the game formed from the $n$ 'th cyclic permutation of $\mathbf{T}_{0}, \mathbf{T}_{n}$, gives us the probability of finding the selected game in each state at times $t=$ $(a+b) i+n$, where $i=0,1,2,3 \ldots$. This is illustrated by Fig. 5. We can calculate the overall probability of a win at time steps $t=(a+b) i+n$ by taking the dot product of

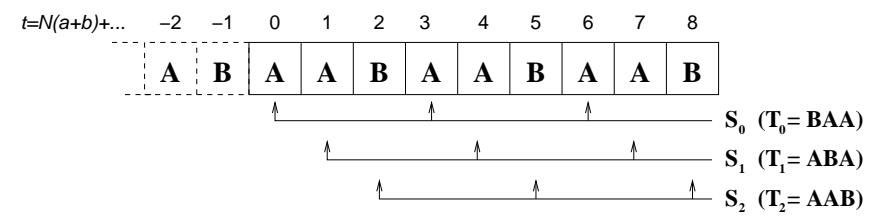

FIG. 5: Illustration of the time steps at which the stationary states $\mathbf{S}_{n}$ of the transition matrices $\mathbf{T}_{n}$ give the probability of finding the combined game in each of the possible states. $N$ is an integer and we take the long time limit (i.e. $N \rightarrow \infty$ ).

the stationary state of the transition matrix $\mathbf{T}_{n}$, with a vector formed from the probabilities of each of the coins from the game played at that time step. These vectors are $\mathbf{p}=\left(\begin{array}{llll}p_{1} & p_{2} & p_{3} & p_{4}\end{array}\right)$ and $\mathbf{q}=\left(\begin{array}{llll}q_{1} & q_{2} & q_{3} & q_{4}\end{array}\right)$, where $\mathbf{p}$ corresponds to $\mathbf{A}$ in Eq. (13) and $\mathbf{q}$ corresponds to $\mathbf{B}$ in Eq. (14). The matrix to the right of the product in $\mathbf{T}_{n}$ corresponds to the game that will be played at time step $t=(a+b) i+n$. Therefore, if the matrix to the right is $\mathbf{A}$ we must take the dot product with $\mathbf{p}$. If it is $\mathbf{B}$, we must take the dot product with $\mathbf{q}$.

An expression for the average probability $P_{\text {win }}$ of a win for the combined game, can thus be found by averaging over all possible cyclic permutations of $\mathbf{T}_{0}$. The gradient, $\operatorname{grad}\left[\left\langle X_{c}(t)\right\rangle\right]$, is then given by Eq. (A1), as before. The resulting expressions are lengthy. Each set of values of $a$ and $b$ yields an expression for $\operatorname{grad}\left[\left\langle X_{c}(t)\right\rangle\right]$ in terms of $\left\{p_{i}\right\},\left\{q_{i}\right\}$, where $i=1,2,3,4$. These expressions are too complicated to set out here explicitly. However, we can numerically plot the analytic equivalent of Figure 4: this is what we have essentially done in Figure 6. The lines show the analytic prediction for the average capital after 500 time steps, $\left\langle X_{c}(500)\right\rangle$, found by multiplying Eq. (A1) by 500. Each line corresponds to a slice through the surface in Fig. 14 at constant $b$. The error bars indicate one standard deviation on the mean over ten ensemble averages of the numerical game. Each ensemble average comprises an average over 50,000 individual runs.

We can see that the numerical and analytic results agree to within one standard deviation. This confirms that the equations generated by the analysis presented in this section are indeed correct. Thus, we have derived expressions for the robustness of the Parrondo effect when two history-dependent games are combined periodically.

\section{VARYING THE SWITCHING PROBABILITY IN THE RANDOM CASE}

We now examine the dependence of the capital on the switching probability in the case that the games are randomly combined. Figure 7 shows the capital after 500 iterations plotted against the probability per iteration $\alpha$ that the game $\left\{p_{i}\right\}$ will be chosen. The curve is symmetric and demonstrates that the capital is greatest if the games are switched with equal probability. When imple- 


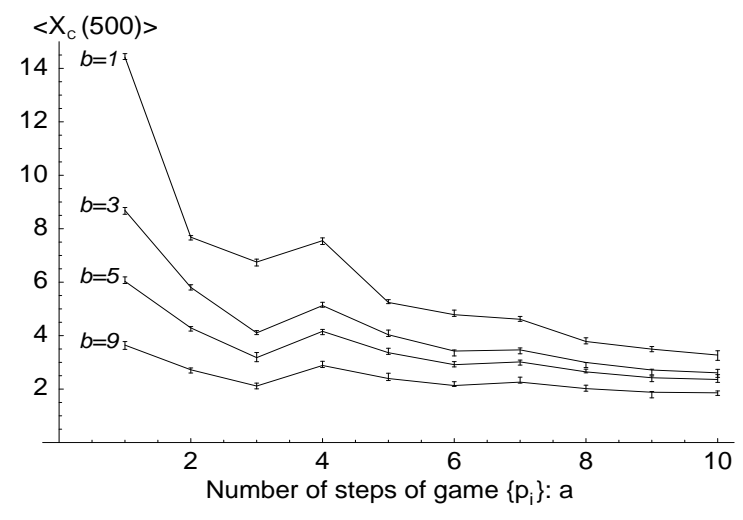

FIG. 6: Comparison of analytic and numerical results for periodic switching. The lines show the analytic predictions at constant $b$, the number of steps of game $\left\{q_{i}\right\}$, for the capital after 500 time steps. The error bars show one standard deviation on the mean over 10 separate ensemble averages, each comprising 50,000 numerical runs.

menting the games it is necessary to assign values to the results of the coin tosses at times $t=-2,-1$ in order to seed the game. This arbitrary choice introduces transients which can slightly bias the final results. However, by allowing the game to first run for 100 iterations, this effect can be eliminated.

The curve in Fig. 7 represents the capital predicted by Eq. (A3) plotted for all $\alpha$ with the same $\left\{p_{i}\right\}$ and $\left\{q_{i}\right\}$. The error bars show one standard deviation either side of the mean capital, averaged over an ensemble of 10 runs. The agreement between the theoretical curve and numerical data is therefore better than one standard deviation. For $\alpha=0$ or 1 we find $\left\langle X_{c}(500)\right\rangle=0$. These values correspond to just playing one $B$ game or the other. Since both games are fair for $\epsilon=0$ the average capital is zero. The fact that the curve is then positive for all values of $\alpha$ means that combining the games with any probability $0<\alpha<1$ leads to a winning combined game.

Parrondo et al showed that combining two losing games could lead to a winning game because switching between the games can break the cycles which cause the "bad" coins to be over-played [1]. We might therefore expect that switching between the games frequently (either by reducing $a$ and $b$ or by choosing a switching probability close to 0.5 ) results in the largest capital gain since this will break up the cycles most effectively. This is indeed confirmed by the results in Fig. 1 and 7 .

\section{SUMMARY}

We have demonstrated that the apparently paradoxical effect of two losing games combining to produce a winning game also applies to combinations of two historydependent games. We derived expressions for the regions of the parameter space in which the effect is observed for

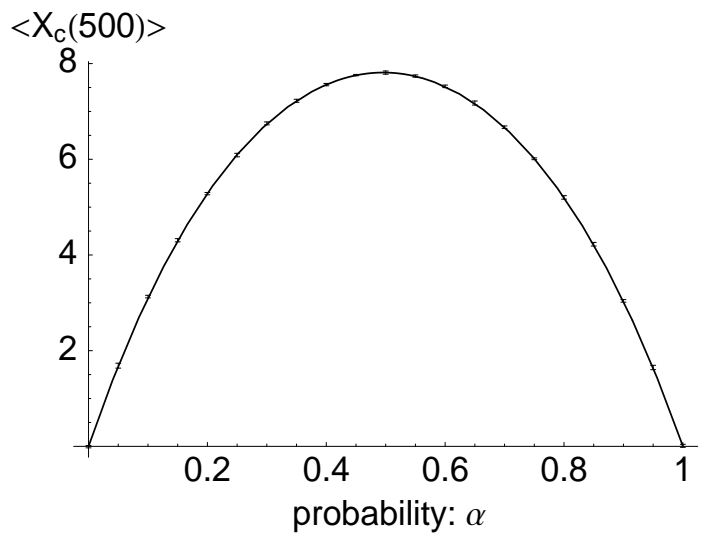

FIG. 7: The value of the capital after 500 games averaged over an ensemble of 500,000 runs. The same games as in Fig. 4 are combined, but this time randomly. Game $\left\{p_{i}\right\}$ is chosen with probability $\alpha$. The solid line shows the theoretical result predicted by Eq. (A3) while the error bars (which are barely visible since they are so small) indicate one standard deviation about the mean capital over an ensemble of 10 runs.

both random and periodic combinations of these games. We derived expressions for the gradient of the average capital and hence the robustness of the Parrondo effect for games combined randomly or periodically.

Our work has therefore expanded the understanding of the Parrondo effect by demonstrating its existence for new combinations of history-dependent games. We are now faced with the more general question as to what property of the constituent games guarantees that the Parrondo effect will be observed. In addition if we were to combine many games of a more general nature than those used to date, how could we predict whether the effect would emerge? We hope that the present work will stimulate further research on such questions, in addition to pursuing applications of the Parrondo effect itself.

\section{Acknowledgments}

We thank Adrian Flitney for helpful comments and suggestions.

\section{APPENDIX A: DERIVATION OF $\epsilon_{\max }$ FOR RANDOM COMBINATIONS OF GAMES}

In this appendix we derive expressions for the maximum value of $\epsilon, \epsilon_{\max }$, for which two games which are fair for $\epsilon=0$ combine to yield a winning game.

Let $P_{\text {win }}$ and $P_{\text {lose }}$ be the probabilities, per iteration for the combined game, that $X_{c}(t)$ will increase or decrease respectively. Then the gradient of the capital line for the combined game $\operatorname{grad}\left[\left\langle X_{c}(t)\right\rangle\right]$ is given by:

$$
\operatorname{grad}\left[\left\langle X_{c}(t)\right\rangle\right]=2 P_{\text {win }}-1 \text {. }
$$


One can derive the following expression for $P_{\text {win }}$ for a game $\left\{r_{i}\right\}$ :

$$
P_{\mathrm{win}}=\frac{r_{1}\left(r_{2}+1-r_{4}\right)}{\left(1-r_{4}\right)\left(2 r_{1}+1-r_{3}\right)+r_{1} r_{2}} .
$$

Substituting for $\left\{r_{i}\right\}$ from Eq. (3) yields:

$$
\begin{aligned}
& \operatorname{grad}\left[\left\langle X_{c}(t)\right\rangle\right]= \\
& \frac{2 \times \ldots}{\left[(1-\alpha) q_{1}+\alpha p_{1}\right]\left[(1-\alpha) q_{2}+\alpha p_{2}\right]+\ldots} \\
& \frac{\ldots\left[(1-\alpha) q_{1}+\alpha p_{1}\right] \times \ldots}{\ldots\left(1-2(\alpha-1) q_{1}+(\alpha-1) q_{3}+2 \alpha p_{1}-\alpha p_{3}\right) \times \ldots} \\
& \frac{\ldots\left[1+(1-\alpha) q_{2}-(1-\alpha) q_{4}+\alpha\left(p_{2}-p_{4}\right)\right]}{\ldots\left[1+(\alpha-1) q_{4}-\alpha p_{4}\right]}-1
\end{aligned}
$$

Now we reintroduce $\epsilon$ via the transformations $p_{i} \rightarrow p_{i}-\epsilon$, $q_{i} \rightarrow q_{i}-\epsilon$ to obtain:

$$
\begin{aligned}
& \operatorname{grad}\left[\left\langle X_{c}(t)\right\rangle\right]= \\
& \frac{2 \times \ldots}{\left[\alpha p_{1}+(1-\alpha) q_{1}-\epsilon\right]\left[\alpha p_{2}+(1-\alpha) q_{2}-\epsilon\right]+\ldots} \\
& \frac{\ldots\left[\alpha p_{1}+(1-\alpha) q_{1}-\epsilon\right] \times \ldots}{\ldots\left[-2 \alpha p_{1}+\alpha p_{3}-2(1-\alpha) q_{1}+(1-\alpha) q_{3}+\epsilon-1\right] \times \ldots} \\
& \quad \ldots\left[\alpha\left(p_{2}-p_{4}\right)+(1-\alpha)\left(q_{2}-q_{4}\right)+1\right] \\
& \ldots\left[\alpha p_{4}+(1-\alpha) q_{4}-\epsilon-1\right]
\end{aligned}
$$

Any games $\left\{p_{i}\right\},\left\{q_{i}\right\}$ defined as above will be losing games for all values of $\epsilon>0$. Thus in order to find the maximum value of $\epsilon$ for which two losing games combine to yield a winning game, we must find the value of $\epsilon$ for which $\operatorname{grad}\left[\left\langle X_{c}(t)\right\rangle\right]=0$. We shall consider games combined with equal probability, therefore $\alpha=\frac{1}{2}$. Setting $\operatorname{grad}\left[\left\langle X_{c}(t)\right\rangle\right]$ equal to zero in Eq. ( $\left.\mathrm{A} 4\right)$ gives:

$$
\begin{array}{r}
\epsilon_{\max }=\frac{-4+p_{1} p_{2}+2 p_{3}+2 p_{4}-p_{3} p_{4}+p_{2} q_{1}+\ldots}{2\left(4+p_{1}+p_{2}-p_{3}-p_{4}+\ldots\right.} \\
\ldots p_{1} q_{2}+q_{1} q_{2}+2 q_{3}-p_{4} q_{3}+2 q_{4}-p_{3} q_{4}-q_{3} q_{4} \\
\left.\ldots q_{1}+q_{2}-q_{3}-q_{4}\right)
\end{array}
$$

Appealing to the condition (Eq. (7)) that $\left\{q_{i}\right\}$ is a fair game for $\epsilon=0$ and in the special case where $q_{2}=q_{3}$ and $p_{2}=p_{3}$, this becomes:

$$
\begin{aligned}
& \epsilon_{\max }= \frac{p_{2}\left[1+q_{2}\left(1+p_{1}-p_{4}\right)-q_{4}\right]+\ldots}{2\left(1-q_{4}\right)+\ldots} \\
& \frac{q_{2}\left[-3+p_{4}\left(2-q_{2}\right)+q_{2}+p_{1} q_{2}+q_{4}\right]}{2 q_{2}\left(3+p_{1}-p_{4}\right)}
\end{aligned}
$$

Similarly in the general case, $\epsilon_{\max }$ is given by:

$$
\begin{aligned}
\epsilon_{\max }= & \frac{p_{2}\left[1-q_{4}+q_{2} p_{1}+q_{3}\left(q_{4}-1\right)\right]+\ldots}{2-2 q_{4}+\ldots} \\
& \frac{\ldots q_{2}\left[-3+p_{4}\left(2-q_{3}\right)+q_{3}+q_{4}\right]+\ldots}{\ldots 2 q_{2}\left(4+p_{1}+p_{2}-p_{3}-p_{4}\right)+\ldots} \\
& \frac{\ldots q_{2}^{2} p_{1}+p_{3} q_{2}\left(2-p_{4}-q_{4}\right)}{\ldots 2 q_{2}\left(q_{2}-q_{3}-q_{4}\right)+2 q_{3}\left(q_{4}-1\right)}
\end{aligned}
$$

[1] J. M. Parrondo, G. P. Harmer, and D. Abbott, Phys. Rev. Lett. 85, 5226 (2000).

[2] P. C. W. Davies, in Physics and life: The Abdus Salam Memorial Lecture, edited by J. Chela-Flores, T. Tobias, and F. Raulin (Kluwer Academic Publishers, 2001), pp. 13-20, in Sixth Trieste Conference on Chemical Evolution, Trieste, Italy.

[3] D. Kinderlehrer and M. Kowalczyk, Arch. Ration. Mech. An. 161, 299 (2002).
[4] D. Heath, D. Kinderlehrer, and M. Kowalczyk, Discrete and continuous dynamical systems-series B 2, 153 (2002)

[5] R. Pyke, Preprint math.PR/0206150, http://xxx.lanl.gov. (2002).

[6] G. P. Harmer and D. Abbott, Chaos 11, 705 (2001).

[7] S. Maslov and Y. Zhang, Int. J. Th. Appl. Finance 1, 377 (1998). 